\title{
Progress Toward Rubella Elimination — Western Pacific Region, 2000-2019
}

\author{
Jennifer K. Knapp, $\mathrm{PhD}^{1}$; Kayla M. Mariano²; Roberta Pastore, $\mathrm{MD}^{2}$; Varja Grabovac ${ }^{2}$; Yoshihiro Takashima, MD, PhD²; James P. Alexander, Jr., MD;
} Susan E. Reef, $\mathrm{MD}^{1}$; José E. Hagan, $\mathrm{MD}^{2}$

Rubella is the leading vaccine-preventable cause of birth defects. Rubella typically manifests as a mild febrile rash illness; however, infection during pregnancy, particularly during the first trimester, can result in miscarriage, fetal death, or a constellation of malformations known as congenital rubella syndrome (CRS), commonly including one or more visual, auditory, or cardiac defects (1). In 2012, the Regional Committee of the World Health Organization (WHO) Western Pacific Region (WPR)* committed to accelerate rubella control, and in 2017, resolved that all countries or areas (countries) in WPR should aim for rubella elimination ${ }^{\dagger}$ as soon as possible $(2,3)$. WPR countries are capitalizing on measles elimination activities, using a combined measles and rubella vaccine, case-based surveillance for febrile rash illness, and integrated diagnostic testing for measles and rubella. This report summarizes progress toward rubella elimination and CRS prevention in WPR during 2000-2019. Coverage with a first dose of rubellacontaining vaccine (RCV1) increased from $11 \%$ in 2000 to $96 \%$ in 2019. During 1970-2019, approximately 84 million persons were vaccinated through 62 supplementary immunization activities (SIAs) conducted in 27 countries. Reported rubella incidence increased from 35.5 to 71.3 cases per million population among reporting countries during 2000-2008, decreased to 2.1 in 2017, and then increased to 18.4 in 2019 as a result of outbreaks in China and Japan. Strong sustainable immunization programs, closing of existing immunity gaps, and maintenance of high-quality surveillance to respond rapidly to and contain outbreaks are needed in every WPR country to achieve rubella elimination in the region.

\footnotetext{
*The Western Pacific Region, one of the six regions of the World Health Organization, consists of 37 countries and areas with a population of approximately 1.9 billion, including American Samoa (United States), Australia, Brunei, Cambodia, China, Cook Islands (New Zealand), Micronesia, Fiji, French Polynesia (France), Guam (United States), Hong Kong (China), Japan, Kiribati, Laos, Macau (China), Malaysia, Marshall Islands, Mongolia, Nauru, New Caledonia (France), New Zealand, Niue (New Zealand), Northern Mariana Islands (United States), Palau, Papua New Guinea, Philippines, Pitcairn Islands (United Kingdom), Samoa, Singapore, Solomon Islands, South Korea, Tokelau (New Zealand), Tonga, Tuvalu, Vanuatu, Vietnam, and Wallis and Futuna (France).

$\dagger$ Rubella elimination is defined as the absence of endemic rubella virus transmission in a defined geographical area (e.g., region or country) for $\geq 12$ months in the presence of a well-performing surveillance system.
}

\section{Immunization Activities}

During 1970-2005, rubella vaccination in $11 \mathrm{WPR}$ countries $\$$ focused on preventing CRS by vaccinating adolescent females; this strategy did not prevent all CRS cases, and countries adopted universal infant immunization (Table 1). By 2000, 16 (44\%) of the 36 WPR countries that report immunization data to $\mathrm{WHO}$ and the United Nations Children's Fund (UNICEF) included RCV1 in the routine immunization schedule; by 2015 , all 36 had introduced it. By 2019, 34 (94\%) countries had included a second dose of rubella-containing vaccine (RCV2). WHO and UNICEF estimate national RCV1 coverage for 27 countries in the region, using annual government-reported survey and administrative data; for the remaining nine countries, 9 coverage data reported by the immunization program are used.

Population immunity of $\geq 85 \%$ is needed to achieve herd immunity and interrupt endemic rubella virus transmission (1). Regional RCV1 coverage increased from $11 \%$ in 2000 to $96 \%$ in 2019 and has been $\geq 90 \%$ since 2015 because of vaccine introduction and achievement of high vaccination coverage in China (2007) and Vietnam (2015) (Figure). In 2019, 24 (67\%) countries achieved $\geq 90 \%$ RCV1 coverage, and $19(53 \%)$ countries achieved $\geq 90 \%$ coverage for RCV1 and RCV2 (Table 1). However, two countries and six islands did not reach $85 \%$ RCV1 coverage, leaving 793,850 infants unprotected.

During 1970-2019, 84.3 million persons were vaccinated during 62 SIAs conducted in 27 countries (weighted regional coverage $=81 \%)($ Table 2$)(4-8)$. Reported administrative coverage was $\geq 95 \%$ in $30(50 \%)$ of 60 SIAs with available data.

\footnotetext{
\$Initial rubella vaccination strategy involved vaccination of adolescent females to prevent CRS in the following countries and areas, years, and age groups: Australia (1971-1994, 12-14 years); Brunei (1978-1995, 12-13 years); Fiji (1975-2005, 11-14 years); French Polynesia (France) (1990s, 10 years); Hong Kong (China) (1978-1995, 11 years); Japan (1977-1995, 12-15 years); Macau (China) (1987-2002, 10-13 years); Malaysia (1987-2008, 12 years); New Zealand (1979-1991, 11 years); Niue (New Zealand) (late 1970s, 11-12 years); Singapore (1976-1982, 11-12 years); and South Korea (1994-2001, 16 years).

T The WHO/UNICEF estimates of national immunization coverage are not calculated for nine areas in the following countries: China (Hong Kong and Macau), France (French Polynesia, New Caledonia, and Wallis and Futuna), New Zealand (Tokelau), and United States (American Samoa, Guam, and Northern Mariana Islands). The Pitcairn Islands (United Kingdom), with a population of $<100$ persons, does not report to $\mathrm{WHO} / \mathrm{UNICEF}$ and is excluded from all calculations.
} 
TABLE 1. Year of introduction, age at vaccination, and estimated coverage with the first and second doses of rubella-containing vaccine (RCV),* and number of confirmed rubella cases ${ }^{\dagger}$ and incidence, ${ }^{\S}$ by country/area- World Health Organization (WHO) Western Pacific Region, 2000, 2010, and 2019

\begin{tabular}{|c|c|c|c|c|c|c|c|c|c|c|c|c|c|}
\hline \multirow[b]{3}{*}{ Country/Area } & \multirow{2}{*}{\multicolumn{2}{|c|}{$\begin{array}{c}\text { Year of } \\
\text { introduction }\end{array}$}} & \multirow{2}{*}{\multicolumn{2}{|c|}{$\begin{array}{c}2019 \text { RCV } \\
\text { schedule, age }\end{array}$}} & \multicolumn{3}{|c|}{2000} & \multicolumn{3}{|c|}{2010} & \multicolumn{3}{|c|}{2019 9ी } \\
\hline & & & & & \multicolumn{2}{|c|}{$\%$ Coverage } & \multirow{2}{*}{$\begin{array}{l}\text { No. of cases } \\
\text { (incidence) }^{\S}\end{array}$} & \multicolumn{2}{|c|}{$\%$ Coverage } & \multirow{2}{*}{$\begin{array}{l}\text { No. of cases } \\
\text { (incidence) }^{\S}\end{array}$} & \multicolumn{2}{|c|}{$\%$ Coverage } & \multirow{2}{*}{$\begin{array}{l}\text { No. of cases } \\
\text { (incidence) }^{\S}\end{array}$} \\
\hline & RCV1 & $\mathrm{RCV} 2$ & 1 st dose & 2nd dose & RCV1 & $\mathrm{RCV} 2$ & & RCV1 & RCV2 & & RCV1 & $\mathrm{RCV} 2$ & \\
\hline Australia** & 1989 & 1992 & $12 m$ & $18 m$ & 91 & $\mathrm{NR}^{\dagger \dagger}$ & $323(15)$ & 94 & 88 & $42(2)$ & $\mathrm{NR}^{+\dagger}$ & 94 & $22(1)$ \\
\hline Brunei** & 1988 & 1996 & $12 m$ & $18 m$ & 99 & 95 & $1(3)$ & 94 & 93 & $1(2)$ & 97 & 98 & $1(2)$ \\
\hline Cambodia & 2012 & 2013 & $9 m$ & $18 \mathrm{~m}$ & $N A^{\S \S}$ & $N A^{\S \S}$ & $\mathrm{NR}^{+\dagger}$ & $N A^{\S \S}$ & $N A^{\S \S}$ & $85(5)$ & 104 & 93 & $30(2)$ \\
\hline China & 2007 & 2010 & $8 m$ & $18 m$ & $N A^{\S \S}$ & $N A^{\S \S}$ & $\mathrm{NR}^{+\dagger}$ & 62 & $62^{919}$ & $43,117(30)$ & 99 & 99 & $32,568(23)$ \\
\hline Hong Kong $(\mathrm{CH})^{* *}$ & 1990 & 1996 & $12 \mathrm{~m}$ & $6 y$ & 100 & 99 & $2,388(343)$ & 95 & 99 & $38(5)$ & $\mathrm{NR}^{+\dagger}$ & 97 & $48(6)$ \\
\hline Japan** & 1989 & 2006 & $12 m$ & $5 y$ & 94 & $N A^{\S \S}$ & $3,123(24)$ & 94 & 97 & $89(1)$ & $97^{\uparrow}$ & $93^{9}$ & $2,306(18)$ \\
\hline Laos & 2011 & 2017 & $9 m$ & $12 m$ & $N A^{\S \S}$ & $N A^{\S \S}$ & $\mathrm{NR}^{+\dagger}$ & $N A^{\S \S}$ & $N A^{\S \S}$ & $31(4)$ & 89 & 63 & $14(2)$ \\
\hline Macau $(\mathrm{CH})^{* *}$ & 1990 & 1994 & $12 m$ & $18 \mathrm{~m}$ & 90 & 89 & $20(37)$ & 92 & 87 & $2(3)$ & 98 & 96 & 79 (122) \\
\hline Malaysia**,*** & 2002 & 2002 & $9 m$ & $12 \mathrm{~m}$ & $N A^{\S \S}$ & $N A^{\S \S}$ & $\mathrm{NR}^{\dagger \dagger}$ & 95 & 95 & $104(3)$ & 97 & 87 & $111(3)$ \\
\hline Mongolia & 2009 & 2009 & $9 m$ & $2 y$ & $N A^{\S \S}$ & $N A^{\S \S}$ & $1,550(570)$ & 97 & 95 & $11(3)$ & 98 & 98 & $5(2)$ \\
\hline New Zealand**,††† & 1990 & 1992 & $15 \mathrm{~m}$ & $4 y$ & 85 & $\mathrm{NR}^{\dagger \dagger}$ & $26(6)$ & 91 & 86 & $2(0)$ & $92^{9}$ & $90^{9}$ & $1(0)$ \\
\hline Papua New Guinea & 2015 & 2015 & $9 m$ & $18 m$ & $N A^{\S \S}$ & $N A^{\S \S}$ & $\mathrm{NR}^{+\dagger}$ & $N A^{\S \S}$ & $N A^{\S \S}$ & $5(1)$ & 33 & 20 & $5(1)$ \\
\hline Philippines & 2010 & 2015 & $9 m$ & $12 m$ & $N A^{\S \S}$ & $N A^{\S \S}$ & $\mathrm{NR}^{+\dagger}$ & $10 \S \S \S$ & $N A^{\S \S}$ & $1,440(14)$ & 73 & 68 & $198(2)$ \\
\hline Singapore** & 1982 & 1990 & $12 m$ & $18 m$ & 96 & 98 & $312(61)$ & 95 & 96 & $158(27)$ & $95^{\natural}$ & $84^{9}$ & $7(1)$ \\
\hline South Korea & 1983 & 1997 & $12-15 \mathrm{~m}$ & $4-6 y$ & 95 & 39 & $107(2)$ & 98 & 98 & $21(0)$ & 97 & 97 & $8(0)$ \\
\hline Vietnam & 2015 & $N A^{\S \S}$ & $18 \mathrm{~m}$ & $N A^{\S \S}$ & $N A^{\S \S}$ & $N A^{\S \S}$ & $\mathrm{NR}^{+\dagger}$ & $N A^{\S \S}$ & $N A^{\S \S}$ & $2,300(24)$ & $90^{\pi}$ & $N A^{\S \S}$ & $69(1)$ \\
\hline \multicolumn{14}{|c|}{ Pacific Island Countries and Territories } \\
\hline American Samoa (US) & $1980 s$ & 2003าศๆๆ & $12 m$ & $4 y$ & 90 & 94 & $0(0)$ & 77 & 65 & $\mathrm{NR}^{+\dagger}$ & $\mathrm{NR}^{+\dagger}$ & $\mathrm{NR}^{+\dagger}$ & $\mathrm{NR}^{+\dagger}$ \\
\hline Cook Islands (NZ) & 2006 & 2006 & $15 \mathrm{~m}$ & $4 y$ & $N A^{\S \S}$ & $N A^{\S \S}$ & $0(0)$ & 99 & 98 & $0(0)$ & 99 ी & 99ी & $0(0)$ \\
\hline $\mathrm{Fiji}^{* *}$ & 2003 & 2004 & $12 \mathrm{~m}$ & $18 \mathrm{~m}$ & $N A^{\S \S}$ & $N A^{\S \S}$ & $\mathrm{NR}^{+\dagger}$ & 94 & 94 & $0(0)$ & $94^{\pi}$ & 94 ๆी & $\mathrm{NR}^{+\dagger}$ \\
\hline French Polynesia $(F R)^{* *}$ & 2010 & 2010 & $12 m$ & $18 m$ & $N A^{\S \S}$ & $N A^{\S \S}$ & $\mathrm{NR}^{+\dagger}$ & 99 & 84 & $0(0)$ & $98^{\natural}$ & $98^{\natural}$ & $\mathrm{NR}^{+\dagger}$ \\
\hline Guam (US) & $1980 \mathrm{~s}$ & 1998 & $12 m$ & $4-6 y$ & 93 & 94 & $0(0)$ & $\mathrm{NR}^{+\dagger}$ & $\mathrm{NR}^{+\dagger}$ & $0(0)$ & $82^{\natural}$ & $83^{\natural}$ & $0(0)$ \\
\hline Kiribati & 2004 & 2007 & $12 m$ & $4 y$ & $N A^{\S \S}$ & $N A^{\S \S}$ & $0(0)$ & 89 & 21 & $0(0)$ & $84^{9}$ & 799 & $0(0)$ \\
\hline Marshall Islands & 1982 & 1998 & $12 m$ & $13 m$ & 93 & 6 & $0(0)$ & 97 & 90 & $0(0)$ & 85 & 64 & $0(0)$ \\
\hline Micronesia & 1982 & 1995 & $12 m$ & $\geq 13 m$ & 85 & 50 & $\mathrm{NR}^{+\dagger}$ & 80 & 75 & $\mathrm{NR}^{+\dagger}$ & 78 & 52 & $0(0)$ \\
\hline Nauru & 2006 & 2006 & $12 m$ & $15 \mathrm{~m}$ & $N A^{\S \S}$ & $N A^{\S \S}$ & $0(0)$ & 99 & 92 & $\mathrm{NR}^{\dagger \dagger}$ & 96 & 96 & $0(0)$ \\
\hline New Caledonia (FR) & 1994 & 1994 & $12 m$ & $16 \mathrm{~m}$ & $\mathrm{NR}^{+\dagger}$ & $\mathrm{NR}^{\dagger \dagger}$ & $\mathrm{NR}^{+\dagger}$ & 99 & 78 & $\mathrm{NR}^{+\dagger}$ & $96^{\natural}$ & $92^{9}$ & $\mathrm{NR}^{+\dagger}$ \\
\hline Niue $(\mathrm{NZ})^{* *}$ & 1979 & 1998 & $15 \mathrm{~m}$ & $4 y$ & 99 & 99 & $0(0)$ & 99 & 99 & $0(0)$ & 100 & 100 & $0(0)$ \\
\hline Northern Mariana Islands (US) & $1980 \mathrm{~s}$ & 1992 & $12 m$ & $4-6 y$ & $N A^{\S \S}$ & $N A^{\S \S}$ & $0(0)$ & 93 & 39 & $0(0)$ & 75 & 90 & $0(0)$ \\
\hline Palau & 1986 & 1995 & $12 \mathrm{~m}$ & $15 \mathrm{~m}$ & 83 & 75 & $0(0)$ & 39 & 39 & $0(0)$ & 97 & 88 & $0(0)$ \\
\hline Samoa & 2003 & 2005 & $12 m$ & $15 \mathrm{~m}$ & $N A^{\S \S}$ & $N A^{\S \S}$ & $\mathrm{NR}^{+\dagger}$ & 56 & 30 & $0(0)$ & 96 & 59 & $0(0)$ \\
\hline Solomon Islands & 2013 & 2018 & $12 m$ & $18 \mathrm{~m}$ & $N A^{\S \S}$ & $N A^{\S \S}$ & $\mathrm{NR}^{+\dagger}$ & $N A^{\S \S}$ & $N A^{\S \S}$ & $0(0)$ & 81 & 55 & $0(0)$ \\
\hline Tokelau (NZ) & 2003 & 2005 & $12 m$ & $15 \mathrm{~m}$ & $N A^{\S \S}$ & $N A^{\S \S}$ & $0(0)$ & 95 & 95 & $0(0)$ & 98 & 98 & $0(0)$ \\
\hline Tonga & 2002 & 2002 & $12 m$ & $18 m$ & $N A^{\S \S}$ & $N A^{\S \S}$ & $0(0)$ & 86 & 84 & $0(0)$ & 99 & 100 & $\mathrm{NR}^{+\dagger}$ \\
\hline Tuvalu & 2005 & 2005 & $12 m$ & $18 \mathrm{~m}$ & $N A^{\S \S}$ & $N A^{\S \S}$ & $0(0)$ & 85 & 87 & $0(0)$ & $88^{\natural}$ & $81^{9}$ & $\mathrm{NR}^{+\dagger}$ \\
\hline Vanuatu & 2015 & $N A^{\S \S}$ & $12 m$ & $N A^{\S \S}$ & $N A^{\S \S}$ & $N A^{\S \S}$ & $\mathrm{NR}^{+\dagger}$ & $N A^{\S \S}$ & $N A^{\S \S}$ & $\mathrm{NR}^{+\dagger}$ & 76 & $N A^{\S \S}$ & $0(0)$ \\
\hline Wallis and Fortuna (FR) & $\mathrm{NR}^{+\dagger}$ & $\mathrm{NR}^{+\dagger}$ & $12 m$ & $16 \mathrm{~m}$ & $N A^{\S \S}$ & $N A^{\S \S}$ & $4(272)$ & $\mathrm{NR}^{+\dagger}$ & $\mathrm{NR}^{+\dagger}$ & $\mathrm{NR}^{+\dagger}$ & 105 & 125 & $\mathrm{NR}^{+\dagger}$ \\
\hline $\begin{array}{l}\text { Total Western Pacific } \\
\text { Region } * * * *\end{array}$ & - & - & - & - & 11 & 11 & $7,854(36)$ & 59 & 59 & $47,446(25)$ & 96 & 91 & $35,472(18)$ \\
\hline
\end{tabular}

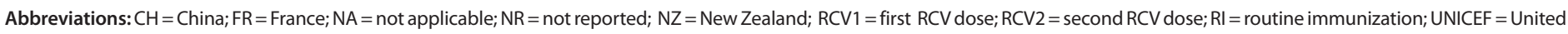
Nations Children's Fund; US = United States.

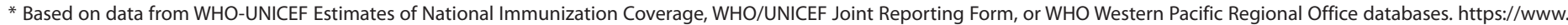
who.int/immunization/monitoring_surveillance/data/en.

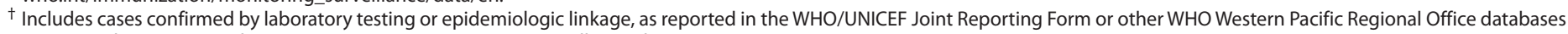
or reports. https://www.who.int/immunization/monitoring_surveillance/data/en.

$\S$ Per million population.

१ 2019 data are as of May 14, 2020; for countries without RCV1 and RCV2 estimates by this date, 2018 coverage values are used.

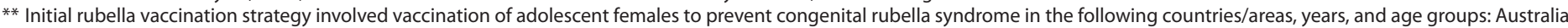

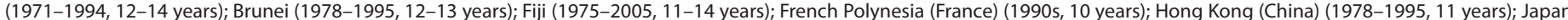

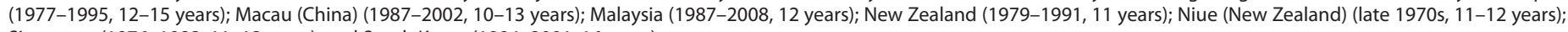
Singapore (1976-1982, 11-12 years); and South Korea (1994-2001, 16 years).

${ }^{+\dagger}$ Not reported because dose was not included in the vaccination schedule for that year.

$\S \S$ Not applicable because country did not report coverage or cases in the year specified.

ๆศ RCV2 coverage as described by Su Q, Ma C, Wen N, et al. https://www.sciencedirect.com/science/article/pii/S0264410X18303499?via\%3Dihub.

*** 2018 RCV schedule includes an additional dose given at age 7 years.

t†† Rubella vaccination of children aged 4 years during 1970-1978, then switch to adolescent female vaccination during $1979-1991$.

$\S \S \S$ RCV2 coverage as described by Lopez AL, Raguindin PFN, Silvestre MA, Fabay XCJ, Vinarao AB, Manalastas R. https://www.hindawi.com/journals/ijpedi/2016/8158712/.

กๆๆ Approximate year of introduction.

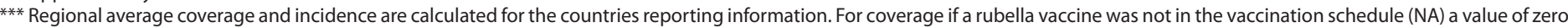
was used, and the country included in the denominator. 
FIGURE. Confirmed rubella cases, ${ }^{*}$ by year of rash onset and country, ${ }^{\dagger}$ and estimated regional coverage with first and second doses of rubellacontaining vaccine ${ }^{\S}$ - World Health Organization (WHO) Western Pacific Region, 2000-2019

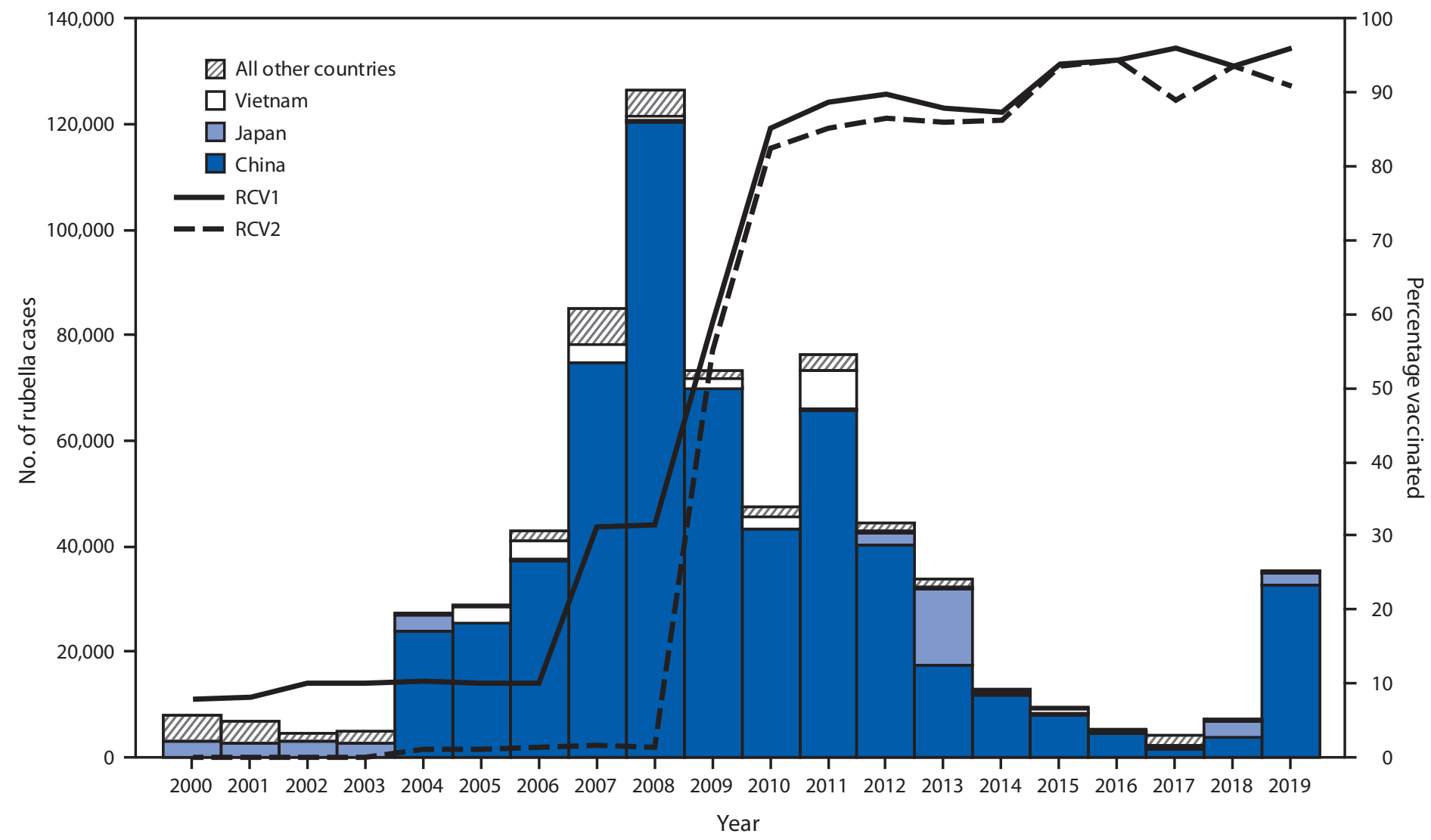

Abbreviations: $\mathrm{RCV} 1$ = first dose of a rubella-containing vaccine; $\mathrm{RCV} 2$ = second dose of a rubella-containing vaccine.

* Confirmed rubella cases reported by countries and areas to WHO. A case of rubella was laboratory-confirmed when rubella-specific immunoglobulin M antibody was detected in serum, rubella-specific RNA was detected by polymerase chain reaction testing, or rubella virus was isolated in cell culture in a person who had not been vaccinated in the 30 days before rash onset; a case of rubella was confirmed by epidemiologic linkage when a case of febrile rash illness was linked in time and place to a laboratory-confirmed rubella case.

† The following countries began reporting rubella surveillance data after 2000: China (2004), Vietnam (2005), Cambodia (2006), Laos (2007), Papua New Guinea (2007), and Malaysia (2010).

$\S$ WHO and United Nations Children's Fund Estimates of National Immunization Coverage, July 15, 2019. https://www.who.int/immunization/monitoring_surveillance/ data/en/.

\section{Surveillance Activities}

Case-based measles and rubella surveillance data are requested monthly by WHO from all WPR countries. Most countries** use an acute fever and maculopapular rash case definition to begin a case investigation and laboratory testing. Some countries also report national or sentinel CRS surveillance data. Rubella cases are confirmed by serology or virus detection or an epidemiologic link to a

\footnotetext{
** As of 2019, 32 countries use a case definition of acute fever and maculopapular rash to identify suspected cases of both rubella and measles, leading to laboratory testing for both diseases. The other four countries have separate surveillance systems for rubella and measles. In those countries, a clinician's diagnosis is based on rubella signs and symptoms (described as maculopapular rash and fever [if measured] and either arthritis/arthralgia or lymphadenopathy) and no testing for measles is done; clinically diagnosed rubella cases are not included in the regional surveillance performance indicators.
}

laboratory-confirmed case. Suspected CRS cases can also be clinically ${ }^{\dagger \dagger}$ confirmed. The WHO Global Measles and Rubella Laboratory Network has supported laboratory confirmation and genotyping since 2005. Indicators of combined measles and rubella surveillance performance include 1) the number of febrile rash illness cases discarded as neither measles nor rubella (target: $\geq 2$ per 100,000 population); 2) the percentage

\footnotetext{
†† CRS can be clinically confirmed in an infant when a qualified physician detects at least two of the complications listed in group A (cataract or cataracts, congenital glaucoma, congenital heart disease, hearing impairment, or pigmentary retinopathy), or one in group A and one in group B (purpura, splenomegaly, microcephaly, developmental delay, meningoencephalitis, radiolucent bone disease, or jaundice within 24 hours after birth).
} 
of cases with adequate investigations that include all essential data elements $\$ \$$ (target: $\geq 80 \%$ ); 3 ) the percentage of cases with adequate blood specimens collected within 28 days of rash onset (target: $\geq 80 \%$, excluding epidemiologically linked cases); and 4) the percentage of specimens with laboratory results reported within 4 days after receipt in the laboratory (target: $\geq 80 \%$ ).

The number of WPR countries reporting rubella data increased from $22(61 \%)$ in 2000 to $29(81 \%)$ in 2019 (Table 1). Five countries, 99 representing $11 \%$ of the regional population, have implemented nationwide CRS surveillance; another seven*** ( $7 \%$ of the population) conduct sentinel surveillance; and four countries ${ }^{\dagger \dagger}$ ( $82 \%$ of the population) and the 21 countries included in the Pacific Islands Countries and Territories ( $<1 \%$ of the population) do not conduct CRS surveillance. During 2010-2018, the median regional nonmeasles/nonrubella discard rate was 3.0 per 100,000 population, ranging from 1.7 (2010) to 9.8 (2018). From 2010 to 2018 , the percentage of suspected measles/rubella cases with adequate investigations increased from $76 \%$ to $84 \%$ and the percentage with adequate blood specimens collected increased from $71 \%$ to $82 \%$; the percentage of specimens with laboratory results increased from $48 \%$ within 7 days to $76 \%$ within 4 days. Regional surveillance indicators are near the target values and all appear to have improved in response to measles outbreaks in 2018.

\section{Rubella Incidence, Outbreaks, and Genotypes}

During 2000-2008, regional rubella incidence increased from 35.5 cases per million population to a peak of 71.3, following initiation of national surveillance in China and Vietnam. Following RCV1 introduction in China, Vietnam, and 18 other countries during 2000-2015, rubella incidence decreased to a historic low of 2.1 per million in 2017 but increased to 18.4 in 2019 (Figure). China, the most populous country, has reported $88 \%$ of regional rubella cases since it began reporting in 2004. Nationwide outbreaks occurred in Hong Kong (2000), the Philippines (2001, 2010, and 2017),

\footnotetext{
$\$ \$$ Essential data elements include name or identifier, date of birth or age, sex, place of residence, vaccination status or date of last vaccination, date of rash onset, date of notification, date of investigation, date of specimen collection, and place of infection or travel history.

Is Countries and areas with nationwide surveillance for congenital rubella syndrome include Australia, Hong Kong (China), Japan, New Zealand, and South Korea.

*** Countries and areas with sentinel-site surveillance for congenital rubella syndrome include Brunei, Cambodia, Laos, Macau (China), Papua New Guinea, Singapore, and Vietnam.

t†† Countries with no CRS surveillance include China, Malaysia, Mongolia, and Philippines.
}

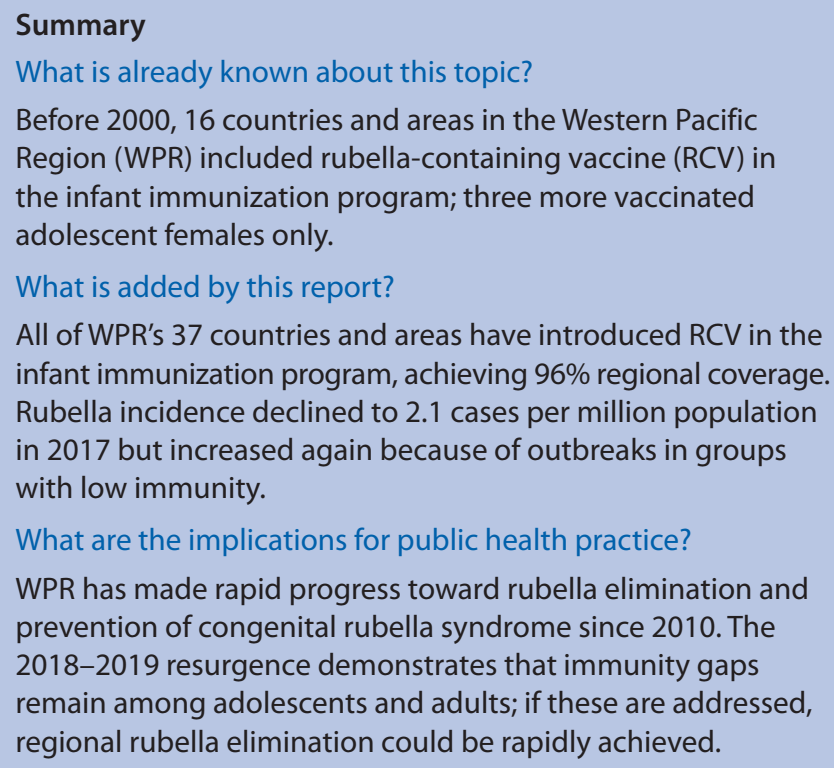

All of WPR's 37 countries and areas have introduced RCV in the infant immunization program, achieving $96 \%$ regional coverage. Rubella incidence declined to 2.1 cases per million population in 2017 but increased again because of outbreaks in groups with low immunity.

What are the implications for public health practice? WPR has made rapid progress toward rubella elimination and prevention of congenital rubella syndrome since 2010. The 2018-2019 resurgence demonstrates that immunity gaps remain among adolescents and adults; if these are addressed, regional rubella elimination could be rapidly achieved.

Samoa (2003), Tokelau (2003), Mongolia (2007), Fiji (2011), Vietnam (2011), Japan (2012-2013), ${ }^{\$ \$ \$}$ Tonga (2002) (Angela Merianos, WHO Pacific Health Security and Communicable Diseases, personal communication, December 2019), and the Solomon Islands (2012) (8). The regional rubella resurgence in 2018-2019 (Figure) was driven by transmission among susceptible males aged 30-55 years in Japan (2018-2019) and among unvaccinated adolescents and young adults in China (2019), with spread to other age groups that included pregnant women. These two outbreaks, which involved rubella virus importations from $>15$ other countries, accounted for $98 \%$ of regional rubella cases in 2018-2019. Only a few countries (Japan, Solomon Islands, and Vietnam) identified CRS cases that occurred after outbreaks. Since 2010, three rubella virus genotypes $(1 \mathrm{E}, 2 \mathrm{~B}$, and $1 \mathrm{~J})$ have been detected in the region. Genotypes 1E and 2B have broad, annual circulation within the region. Genotype 1J was detected in four WPR countries before 2013, but not since.

\section{Regional Verification of Rubella Elimination}

The Western Pacific Regional Committee (1) urged countries to submit measles elimination progress reports for review by the Regional Verification Commission in 2013; verification guidelines were revised in 2017 to include verification of rubella elimination (1). As of September 2019, five of seventeen

$\overline{\$ S \$}$ https://apps.who.int/immunization_monitoring/globalsummary/timeseries/ tsincidencerubella.html. 
TABLE 2. Characteristics of nationwide rubella supplementary immunization activities (SIAs), ${ }^{*}$ by year and country/area — World Health Organization (WHO) Western Pacific Region, 1970-2019 ${ }^{\dagger}$

\begin{tabular}{|c|c|c|c|c|c|}
\hline Country/Area & Year & RCV used & SIA type & Age group targeted & $\begin{array}{c}\text { Population reached in } \\
\text { targeted age group } \\
\text { no. }(\%)\end{array}$ \\
\hline American Samoa (US) & 2019 & MMR & M-outbreak & $6 \mathrm{~m}$-adults & $12,932(41)$ \\
\hline Australia & 1998 & MMR & Catch-up & $\begin{array}{l}1-3.5 y \\
5-12 y\end{array}$ & $\begin{array}{r}60,028(37) \\
1,333,980(75)\end{array}$ \\
\hline Brunei & $2008-2009$ & MMR & Catch-up & $3-6 y$ & 27,161 (98) \\
\hline \multirow[t]{3}{*}{ Cambodia } & 2013 & MR & Catch-up & $9 m-14 y$ & $4,576,633(105)^{\S}$ \\
\hline & 2016 & MR & M-outbreak & $9 m-4 y$ & 766,743 (91) \\
\hline & 2017 & $M R$ & Follow-up & $6 m-4 y$ & $1,451,821(90)^{9}$ \\
\hline Cook Islands (NZ) & 2006 & MR & Catch-up & $\begin{array}{c}1-15 y \\
F: 16-35 y\end{array}$ & $5,829(90)$ \\
\hline \multirow{4}{*}{ Fiji } & 2006 & MR & M-outbreak & $6 m-4 y$ & 89,747 (98) \\
\hline & 2017 & $\mathrm{MR}$ & Catch-up & $1-11 y$ & 178,069 (95) \\
\hline & 2019 & MR & M-outbreak & $6 m-4 y$ & $85,911(100)$ \\
\hline & & & & $19 y-39 y$ & $257,566(94)$ \\
\hline Hong Kong $(\mathrm{CH})$ & 1997 & MMR & Catch-up & $19-39 y$ & $1,100,464(77)$ \\
\hline \multirow[t]{4}{*}{ Kiribati } & 2006 & $\mathrm{MR}$ & Catch-up & $\begin{array}{c}1-14 y \\
F: 15-19 y\end{array}$ & $40,568(95)$ \\
\hline & 2009 & MMR & Follow-up & $1-4 y$ & $9,865(107)^{\S}$ \\
\hline & 2013 & $\mathrm{MR}$ & Follow-up & $1-4 y$ & $1,700(85)$ \\
\hline & 2019 & MR & Catch-up & $1-14 y$ & $42,838(107)^{\S}$ \\
\hline \multirow[t]{4}{*}{ Laos } & 2011 & MR & M-outbreak & $9 m-19 y$ & $2,614,002(97)$ \\
\hline & 2014 & MR & M-outbreak & $9 m-9 y$ & $1,569,224(100)$ \\
\hline & 2017 & MR & Follow-up & $9 m-4 y$ & $703,924(100)$ \\
\hline & 2019 & MR & M-outbreak & $6 m-9 y$ & $937,064(60)$ \\
\hline Malaysia & 1987-1989 & Rubella & Catch-up & F: $15-44 y$ & NR (62) \\
\hline \multirow[t]{3}{*}{ Marshall Islands } & 2002 & MMR & Follow-up & $1-4 y$ & $4,383(77)$ \\
\hline & 2003 & MMR & M-outbreak & $6 m-40 y$ & $37,111(91)$ \\
\hline & 2019 & $\mathrm{MR}$ & M-outbreak & $1-5 y$ & NR (79) \\
\hline Micronesia & 2014 & MMR & M-outbreak & $6 m-49 y$ & $71,388(87)$ \\
\hline \multirow[t]{3}{*}{ Mongolia } & 2012 & MR & Catch-up & $3-14 y$ & $522,429(93)$ \\
\hline & 2016 & MR & M-outbreak & $18-30 y$ & $549,846(88)$ \\
\hline & 2019 & $\mathrm{MR}$ & Catch-up & $10-18 y$ & $400,961(96)$ \\
\hline \multirow[t]{3}{*}{ New Zealand } & 1970 & Rubella & Catch-up & $5-9 y$ & NR (95) \\
\hline & 1997 & MMR & M-outbreak & $2-10 y$ & $474,022(75)$ \\
\hline & 2001 & MMR & Catch-up & $5-10 y$ & NR (NR) \\
\hline Niue (NZ) & 2003 & MMR & Catch-up & $5-11 y$ & $100(36)$ \\
\hline \multirow[t]{3}{*}{ Northern Mariana Islands (US) } & 2002 & MMR & Follow-up & $1-6 y$ & $438(35)$ \\
\hline & 2018 & MR & Catch-up & $1-18 y$ & $36,175(74)$ \\
\hline & 2019 & MR & Catch-up & $19-62 y$ & NR (74) \\
\hline \multirow[t]{2}{*}{ Papua New Guinea } & 2015-2016 & MR & M-outbreak & $6 m-15 y$ & $1,238,290(63)$ \\
\hline & 2019 & MR & Follow-up & $6 m-4 y$ & $1,180,422(101)^{\S}$ \\
\hline \multirow[t]{4}{*}{ Philippines } & 2011 & MR & M-outbreak & $9 m-8 y$ & $15,649,907(84)$ \\
\hline & 2014 & $\mathrm{MR}$ & M-outbreak & $9 m-4 y$ & $10,402,489(91)$ \\
\hline & 2018 & MMR & M-outbreak & $6 m-4 y$ & 4,982,898 (46) \\
\hline & 2019 & MMR & M-outbreak & $5-12 y$ & $2,457,514(29)$ \\
\hline
\end{tabular}

See table footnotes on page 749.

(29\%) countries 999 (Australia, Brunei, Macau, New Zealand, and South Korea) have been verified to have achieved and sustained rubella elimination (9).

\footnotetext{
999 The Regional Verification Commission reviews measles and rubella elimination reports from 17 units: each of $14 \mathrm{WHO}$ member states, two Chinese Special Administrative Regions (Hong Kong and Macau), and the subregion of the Pacific Island Countries and Territories. The Pacific Islands Countries and Territories are reviewed as a single epidemiologic unit; they include American Samoa (United States), Cook Islands, Fiji, French Polynesia (France), Guam (United States), Kiribati, Marshall Islands, Micronesia, Nauru, New Caledonia (France), Niue (New Zealand), Northern Mariana Islands (United States), Palau, Samoa, Solomon Islands, Tokelau (New Zealand), Tonga, Tuvalu, Vanuatu, and Wallis and Futuna (France).
}

\section{Discussion}

Following the $2012 \mathrm{WHO}$ Regional Committee resolution for rubella control, introduction of combined measles and rubella vaccine accelerated, and nearly all countries in WPR now include $2 \mathrm{RCV}$ doses in the routine immunization program. Regional coverage is high, and rubella incidence declined to a historic low in 2017.

Despite high regional coverage, variation in immunity exists among and within countries. Eight countries were unable to reach protective herd immunity of $85 \%$ in their 2018 birth 
TABLE 2. (Continued) Characteristics of nationwide rubella supplementary immunization activities (SIAs), ${ }^{*}$ by year and country/area — World Health Organization (WHO) Western Pacific Region, 1970-2019 ${ }^{\dagger}$

\begin{tabular}{|c|c|c|c|c|c|}
\hline Country/Area & Year & RCV used & SIA type & Age group targeted & $\begin{array}{c}\text { Population reached in } \\
\text { targeted age group } \\
\text { no. }(\%)\end{array}$ \\
\hline \multirow[t]{6}{*}{ Samoa } & 2003 & MR & R-outbreak & $\begin{array}{c}1-18 y \\
F: 19-49 y\end{array}$ & $\begin{array}{c}47,448(88) \\
19,730(103)^{\S}\end{array}$ \\
\hline & 2005 & MR & Follow-up & $9 m-2 y$ & $11,610(86)$ \\
\hline & 2008 & MR & Follow-up & $9 m-4 y$ & $22,864(91)$ \\
\hline & 2009 & MR & Disaster & $6 m-4 y$ & $21,142(76)$ \\
\hline & 2017 & MR & M-outbreak & $1-12 y$ & $57,229(95)$ \\
\hline & 2019 & MR & M-outbreak & $6 m-50 y$ & $187,369(93)$ \\
\hline \multirow[t]{2}{*}{ Singapore } & 1997 & MMR & M-outbreak & $12-18 y$ & NR (NR) \\
\hline & 2013 & MMR & Catch-up & $6-7 y$ & $38,436(95)$ \\
\hline \multirow[t]{3}{*}{ Solomon Islands } & 2012 & MR & R-outbreak & $1-4 y$ & $67,106(101)^{\S}$ \\
\hline & 2014 & $\mathrm{MR}$ & M-outbreak & $6 m-29 y$ & $394,584(105)^{\S}$ \\
\hline & 2019 & MR & M-outbreak & $6 m-5 y$ & $87,855(99)$ \\
\hline \multirow[t]{2}{*}{ South Korea } & 2001 & MR & M-outbreak & $8-16 y$ & $5,614,327(96)$ \\
\hline & $2006-2009$ & MMR & Follow-up & $8 y$ & $2,205,333(99)$ \\
\hline \multirow[t]{2}{*}{ Tokelau (NZ) } & 2003 & MMR & R-outbreak & $1-15 y$ & $838(98)$ \\
\hline & & & & $\mathrm{F}: \mathrm{CBA}^{* *}$ & \\
\hline \multirow[t]{3}{*}{ Tonga } & 2002 & MR & R-outbreak & $1-13 y$ & $37,279(95)$ \\
\hline & & & & $F: 14-40 y$ & $18,321(95)$ \\
\hline & 2019 & MR & M-outbreak & $6 m-24 y$ & $54,590(94)$ \\
\hline \multirow[t]{2}{*}{ Tuvalu } & 2005 & MR & Catch-up & $1-34 y$ & $5,469(96)$ \\
\hline & 2010 & MR & Follow-up & $1-5 y$ & $1,095(79)$ \\
\hline \multirow[t]{2}{*}{ Vanuatu } & 2013 & $\mathrm{MR}$ & Follow-up & $1-4 y$ & $33,604(102)^{\S}$ \\
\hline & 2015 & MR & Catch-up & $1-15 y$ & $103,676(103)^{\S}$ \\
\hline \multirow[t]{2}{*}{ Vietnam } & 2014-2015 & MR & Catch-up & $1-14 y$ & $19,735,753(98)$ \\
\hline & 2016 & MR & Catch-up & $16-17 y$ & $1,787,588(95)$ \\
\hline Total Western Pacific Region & 1970-2019 & - & - & - & $84,339,251(81)$ \\
\hline
\end{tabular}

Abbreviations: $\mathrm{CBA}=$ childbearing age; $\mathrm{CH}=\mathrm{China} ; \mathrm{F}=$ female; $\mathrm{FR}=$ France; $\mathrm{m}=$ months; $\mathrm{M}$-outbreak = measles outbreak; $\mathrm{MMR}=$ measles, $\mathrm{mumps}$, and rubella vaccine; $\mathrm{MR}=$ measles and rubella vaccine; $\mathrm{NR}=$ not reported; $\mathrm{NZ}=$ New Zealand; $\mathrm{R}$-outbreak = rubella outbreak; $\mathrm{RCV}=$ rubella-containing vaccine; $\mathrm{SI} A=$ supplemental immunization activity; $U S=$ United States; $y=$ years.

* Rubella SIAs use a combined measles-rubella vaccine; these SIAs generally use two target age ranges: 1) initial, nationwide catch-up SIAs target all children aged 9 months-14 years, with the goal of eliminating susceptibility to rubella virus in the general population, and 2) follow-up nationwide SIAs generally conducted every 2-4 years target children not included in the previous SIA, who are generally aged 9-59 months (their goal is to protect children who did not respond to the first measles vaccine dose and to provide another opportunity for vaccination). Rubella SIAs also occur as a result of measles outbreak response SIAs when MR or MMR is used for the campaign. The exact age range for follow-up or outbreak SIAs depends on the age-specific incidence of measles, coverage with vaccine containing measles and rubella through routine services, and the time since the last SIA.

† SIAs conducted in 2019 might display interim rather than final numbers of persons vaccinated.

$\S$ Values $>100 \%$ indicate that the intervention reached more persons than the estimated target population. The numerator was the total children vaccinated, and the denominator was the estimated target calculated for vaccination.

I A post-campaign coverage survey estimated that $75 \%$ of children within the targeted ages were vaccinated.

** The SIA denominator indicates that >15 birth cohorts were targeted during this rubella outbreak response; it is expected that, similar to what was found for SIAs on other islands with rubella outbreaks at that time, the additional vaccine recipients were women of childbearing age.

cohorts, perpetuating immunity gaps among children. Recent success achieving high coverage also masks susceptibility among older persons. In WPR, immunity gaps developed from historical adolescent female vaccination programs and by introduction of rubella vaccine in the childhood immunization program without vaccinating those who were not age-eligible according to the childhood vaccination schedule at the time of introduction. As long as immunity gaps persist, countries remain vulnerable to importations, outbreaks that include adults, and CRS-affected pregnancies. Lack of coordination toward elimination among countries and regions creates an inequitable strain on achieving and maintaining rubella elimination because of importations via travel and transit.
Strategies to close identified immunity gaps vary by country. Japan is targeting adult males, testing for immunity and vaccinating susceptible persons. Vietnam annually targets children in a portion of districts determined to be at high risk. Other countries have incidentally boosted immunity to rubella by conducting SIAs in response to measles outbreaks, using combined measles-rubella or measles-mumps-rubella vaccine, although rarely in response to rubella outbreaks.

The World Bank classifies 10 countries in the region as lowmiddle income, ${ }^{* * * *}$ allowing some opportunities for external support for the routine immunization program, targeted

**** Cambodia, Kiribati, Laos, Micronesia, Mongolia, Papua New Guinea, Philippines, Solomon Islands, Vanuatu, and Vietnam. 
immunization activities, and outbreak response support. However, external immunization funding is not currently well-aligned with strategies to achieve a regional elimination goal. The remaining countries must self-finance rubella elimination, given the absence of a broad mechanism for external immunization funding support in middle income countries. In addition, many countries use domestic vaccine suppliers that set vaccine prices and whose production capacity might not meet outbreak response needs. Five countries have been verified as having eliminated endemic rubella transmission; however, other countries with a long history of rubella vaccination and surveillance and with a low annual incidence might also have achieved elimination but have not yet requested verification.

The findings in this report are subject to at least three limitations. First, sensitivity of integrated measles and surveillance for rubella is low because it is a milder illness, resulting in underdetection of cases. Second, direct comparisons among countries might not be valid because of variations in capacity for case investigation and laboratory testing, the monitoring of progress toward elimination, level and source of financing, and the priority given to closing immunity gaps. Finally, the region has countries with widely disparate population sizes, and regional trends might obscure challenges or successes in less populous countries.

The participation of all WPR countries will be needed to attain regional rubella elimination and prevent the devastating consequences of rubella infection during pregnancy. Efforts to achieve these goals include sustaining high population immunity, identifying and addressing existing immunity gaps, and maintaining high-quality surveillance to allow for rapid outbreak detection and prompt response to contain outbreaks.

Corresponding author: Jennifer K. Knapp, JKnapp1@cdc.gov, 404-718-6353.

${ }^{1}$ Global Immunization Division, Center for Global Health, CDC; ${ }^{2}$ Expanded Programme on Immunization, World Health Organization Western Pacific Regional Office, Manila, Philippines.
All authors have completed and submitted the International Committee of Medical Journal Editors form for disclosure of potential conflicts of interest. No potential conflicts of interest were disclosed.

\section{References}

1. World Health Organization Regional Committee for the Western Pacific. Regional strategy and plan of action for measles and rubella elimination in the Western Pacific. Manila, Philippines: World Health Organization Regional Committee for the Western Pacific; 2018. https://iris.wpro.who. int/bitstream/handle/10665.1/14227/9789290618515-eng.pdf

2. World Health Organization Regional Committee for the Western Pacific. Resolution WPR/RC63.R5: elimination of measles and acceleration of rubella control. Manila, Philippines: World Health Organization Regional Committee for the Western Pacific; 2012. https://iris.wpro.who.int/ bitstream/handle/10665.1/8025/WPR_RC063_Res05_2012_en.pdf

3. World Health Organization Regional Committee for the Western Pacific. Resolution WPR/RC68.R1: measles and rubella elimination. Manila, Philippines: World Health Organization Regional Committee for the Western Pacific; 2017. https://iris.wpro.who.int/bitstream/ handle/10665.1/13717/WPR-RC068-Res01-2017-en.pdf

4. Hagan JE, Kriss JL, Takashima Y, et al. Progress toward measles elimination-Western Pacific Region, 2013-2017. MMWR Morb Mortal Wkly Rep 2018;67:491-5. https://doi.org/10.15585/mmwr.mm6717a3

5. Chuang SK, Lau YL, Lim WL, Chow CB, Tsang T, Tse LY. Mass measles immunization campaign: experience in the Hong Kong Special Administrative Region of China. Bull World Health Organ 2002;80:585-91.

6. Ho HJ, Low C, Ang LW, et al. Progress towards measles elimination in Singapore. Vaccine 2014;32:6927-33. https://doi.org/10.1016/j. vaccine. 2014.10 .046

7. Gilbert GL, Escott RG, Gidding HF, et al. Impact of the Australian Measles Control Campaign on immunity to measles and rubella. Epidemiol Infect 2001;127:297-303. https://doi.org/10.1017/ S0950268801005830

8. Durski KN, Tituli C, Ogaoga D, et al. An outbreak investigation of congenital rubella syndrome in Solomon Islands, 2013. Western Pac Surveill Response J 2016;7:10-3. https://doi.org/10.5365/ wpsar.2015.6.4.005

9. World Health Organization Regional Office for the Western Pacific. Meeting report of the eighth annual meeting of the Regional Verification Commission for measles and elimination in the Western Pacific September 16-20, 2019; Hanoi, Vietnam. https://iris.wpro.who.int/ handle/10665.1/14458 